\title{
Privação de Sono e Exercício Físico
}

\section{Sleep Deprivation and Exercise}

Hanna Karen M. Antunes ${ }^{1,3}$

Monica L. Andersen²

Sergio Tufik

Marco Tulio De Mello, 2,3

1. Departamento de Biociências, Universidade Federal de São Paulo, UNIFESP

2. Departamento de Psicobiologia, Universidade Federal de São Paulo, UNIFESP

3. Centro de Estudos em

Psicobiologia e Exercício-CEPE

Endereço para correspondência: R. Marselhesa, 535 - Vila Clementino São Paulo- SP - CEP 04020-060 e-mail: hanna.karen@unifesp.br

Submetido em 02/05/2007

Versão final recebida em 22/05/2007 Aceito em 03/07/2007

\section{RESUMO}

A privação do sono é a remoção ou supressão parcial do sono, e esta condição pode causar diversas alterações: endócrinas, metabólicas, físicas, cognitivas, neurais e modificações na arquitetura do sono, que em conjunto comprometem a saúde e a qualidade de vida do sujeito nestas condições. Já o exercício físico praticado regularmente promove benefícios como melhora do aparato cardiovascular, respiratório, endócrino, muscular e humoral, além disso, pode melhorar a qualidade do sono. Entretanto, a associação desses dois parâmetros não tem sido bem explorada, em parte pela dificuldade conseguir voluntários que se submetam a essa condição principalmente sem nenhum tipo de compensação financeira. A maioria dos estudos que investigaram o binômio exercício físico e privação de sono focou os efeitos no desempenho aeróbio. Embora ainda haja controvérsias, os estudos apontam para pequena ou nenhuma alteração desse parâmetro quando as duas situações se fazem presentes. Em relação à potência anaeróbia e força não tem sido encontrados alterações significativas, mas para eventos prolongados, parece haver uma interação entre a privação de sono e o exercício físico, o que sugere um mecanismo de proteção. Entretanto, é importante considerar que uma das alterações mais importantes causadas pela privação do sono é o aumento na percepção subjetiva, que por si só já representa um fator para diminuição e comprometimento do desempenho físico e pode representar um elemento de "mascaramento" dos efeitos deletérios da privação. Assim, o objetivo da presente revisão é o de discutir os diferentes aspectos da relação entre o exercício físico e a privação de sono, evidenciando seus efeitos e reflexos no desempenho físico.

Palavras-chave: privação do sono, exercício físico, sono.

\begin{abstract}
Sleep deprivation can be defined as total or partial suppress of sleep and is associated with alterations in endocrine, metabolic, physical, cognitive functions and modifications of the sleep patterns that compromise health and quality of life. Physical exercise is associated with improvement of cardiovascular, respiratory, muscular, endocrine and nervous system, and a better sleep quality. However, the association of these two conditions is unclear, partly due to the difficulty to obtain volunteers to participate in this type of protocol with no financial compensation. The majority of the studies which investigate the association between physical exercises and sleep deprivation focus on aerobic performance and verify little or no effect of this parameter. Concerning anaerobic power and strength, significant alterations have not been found; however, for prolonged events there may be an interaction between these two factors, which suggests a protection mechanism. Nevertheless, it is important to consider that one of the main alterations caused by sleep deprivation the increase of the subjective perception, which presents a factor to decrease and compromise the physical performance per se, and may represent a masking element of the deleterious effects of sleep deprivation. Thus, the aim of present review is to discuss the different aspects of relationship between physical exercise and sleep deprivation, showing their effects and consequences in physical performance.
\end{abstract}

Keywords: sleep deprivation, physical exercise, sleep.

\section{INTRODUÇÃO AO SONO E À PRIVAÇÃO DE SONO}

Sabe-se que o sono é um estado vital e complexo caracterizado por processos ativos e altamente organizados ${ }^{(1)}$, que pode ser distribuído em distintos estágios: sono NREM (Non-Rapid Eye Movements) e sono REM (Rapid Eye Movements) ${ }^{(2,3)}$. O sono NREM pode ser subdivido em quatro estágios (ou fases) 1 a 4, correspondendo à profundidade do sono, e a presença de marcadores eletrofisiológicos específicos, sendo os estágios 3 e 4 também chamados de sono delta ou de ondas lentas (SWS- slowwave sleep) $^{(4)}$ (para melhor revisão ver Martins et al., 2004 $4^{(5)}$ ).

A remoção parcial ou supressão do sono em um organismo é conhecida como privação de sono ${ }^{(6)}$. Historicamente, estudos sobre a privação de sono tiveram início em 1894 com os experimentos de De Manacéine ${ }^{(7)}$, onde foi demonstrado que, filhotes e cães adultos, morriam após alguns dias de privação de sono, e que essa privação causava severas lesões no sistema nervoso central desses animais ${ }^{(8)}$.

Em humanos, o primeiro estudo foi conduzido por Patrick e Gilbert $^{(9)}$ (1896). Os autores privaram de sono indivíduos jovens de 88 a 90 horas, e observaram prejuízos no tempo de reação, na habilidade motora voluntária e na habilidade em memorizar. Ao término do experimento, os voluntários dormiram por 10,5 a 12 horas e recuperaram a sua condição basal após acordar. Kleitman ${ }^{(10)}$ (1920) observou que sujeitos privados de sono apresentavam menos sono na manhã seguinte do que durante o meio da noite de privação de sono. Baseado nos resultados observados, ele afirmou que outras influências poderiam nortear a natureza da sonolência, uma vez que foram encontradas modificações desse comportamento nos diferentes horários do dia. Desta forma, ele também sugeriu que durante a vigília prolongada, o tempo de reação pode não variar propondo que esta condição poderia não causar um efeito deletério na circuitaria neural. 
Anos mais tarde foram realizados alguns relatos do uso da privação de sono como elemento de tortura durante a guerra da Coréia. Esses relatos lançaram a idéia de que a privação prolongada de sono poderia levar a alteração mental e deterioração física. Williams e colaboradores ${ }^{(11)}(1959)$ reportaram que homens alistados no exército apresentavam prejuízos no seu desempenho após um período de privação de sono agudo. Esse resultado levou a conclusão de que o prejuízo no desempenho ocorreu principalmente devido a lapsos descritos como "breves períodos de não responsividade acompanhados por uma sonolência extrema e por um declínio na amplitude do alfa EEG (eletroencefalograma)". Não foram observados sinais ambíguos de psicose durante ou após o período da privação do sono.

Outro estudo clássico envolvendo um período longo de privação de sono foi conduzido por pesquisadores da Universidade de Oklahoma, que monitoraram a vigília prolongada de um Disc Jockey de New York. Peter Tripp permaneceu acordado por 201 horas devido a estímulos constantes. Com poucos dias de privação de sono, houve relatos de alucinações, ele dizia que via ratos, gatos e teias de aranha, além de ter apresentado paranóia insistindo que havia a presença de um eletricista que havia colocado eletrodos em seus pés. Na mesma época, o Guinness Book of World Records atestou que o recorde de permanência em vigília foi de 260 horas, no entanto, um cuidadoso monitoramento desse feito não foi bem documentado. Em 1964, um aluno de 17 anos do segundo grau decidiu, para estudar os efeitos da ausência de sono para um projeto de ciências na escola e para quebrar o recorde mundial, privar-se de sono. Após 264 horas (11 dias) de privação de sono, Randy Gardner foi conduzido ao hospital Naval americano onde seu sono foi continuamente registrado por EEG, EOG (eletrooculograma) e EMG (eletromiograma) após o período de privação e durante as semanas seguintes. Na primeira recuperação de sono ele dormiu por 14 horas e 40 minutos, mas devido aos escassos conhecimentos da época, a quantificação exata do sono adicional durante a recuperação do sono não foi considerada relevante. Assim, nenhum efeito foi observado e ele não apresentou sinais de psicose ${ }^{(12)}$.

O primeiro experimento de privação seletiva de sono foi realizado por Dement ${ }^{(13)}$ (1960). O autor fez uma privação seletiva do sono REM e demonstrou que, a privação desta fase do sono, causava um aumento do percentual do sono REM durante o período de recuperação em relação ao seu basal, denominando esse fenômeno de efeito rebote. Além disso, ele observou que poderiam ocorrer distúrbios psicológicos, concluindo que a supressão dos sonhos poderia desencadear uma séria disfunção da personalidade. A existência de um rebote de sono após um período de privação ${ }^{(14)}$, demonstra que o sono não é um simples período de redução da atividade ou do alerta regulado pelo ritmo circadiano ou ultradiano ${ }^{(15)}$.

No Brasil, o Grupo do Sono do Departamento de Psicobiologia da UNIFESP, ao longo dos anos, tem publicado diversos estudos com a temática da privação de sono. O trabalho pioneiro realizado por Tufik e colaboradores $^{(16)}$ (1978) demonstrou que animais privados de sono REM foram mais responsivos à apomorfina do que os controles nãoprivados (grupo controle), sugerindo que esse tipo de privação de sono induzia a uma supersensibilidade dos receptores dopaminérgicos no cérebro. Na continuidade deste trabalho, o autor demonstrou que a agressividade observada nos ratos privados de sono REM, mostrada pela apomorfina, estava relacionada com uma supersensibilidade dos receptores dopaminérgicos pós-sinápticos ${ }^{(17)}$, o que o levou a questionar se essa supersensibilidade não estaria relacionada a um turnover dopaminérgico ${ }^{(18)}$. Mais tarde Nunes Jr e colaboradores ${ }^{(19,20)}$ observaram o aumento dos receptores dopaminérgicos D2 com a privação do sono REM, sugerindo uma supersensibilidade desse tipo de receptor, e que este fenômeno poderia explicar as mudanças previamente relatadas por Tufik em 1978 ${ }^{(16)}$.

Além disso, o trabalho inicial de Tufik ${ }^{(16)}$ (1978) permitiu a realização de inúmeros outros estudos relacionando não apenas o sistema dopaminérgico, mas também o colinérgico(21-23).

Ainda nesta linha de privação do sono REM, D'Almeida ${ }^{(24)}$ colaboradores (1997), utilizando a técnica da privação de sono com plataforma única, não encontraram associações desse procedimento com o estresse oxidativo em ratos, sugerindo que as alterações observadas poderiam ser atribuídas a fatores como a imobilização e o isolamento social presente nessa técnica. Um ano mais tarde foi feita a descrição de uma redução significativa dos níveis de glutationa no tálamo e no hipotálamo, sugerindo que áreas específicas do cérebro podem ser diferencialmente susceptíveis aos efeitos do estresse oxidativo com a privação de sono(25).

O Grupo de Sono ainda tem conduzido diversos outros trabalhos relacionados aos efeitos da privação do sono no eixo hipotálamo-hipófise-adrenal(26,27), nos níveis plasmáticos das endotelinas( ${ }^{(28)}$, na memória ${ }^{(29)}$ e na atenção visual(30). Atualmente, uma linha de pesquisa promissora do Departamento de Psicobiologia tem investigado os efeitos da privação de sono REM associados ao uso da cocaína na ereção peniana e na ejaculação de ratos. Os trabalhos têm demonstrado a existência de importantes alterações hormonais devido à privação de sono, sendo a redução da testosterona o seu resultado mais marcante ${ }^{(31)}$.

Considerando a complexidade e a importância do tema, o objetivo da presente revisão é o de discutir os diferentes aspectos da relação entre o exercício físico e a privação de sono, evidenciando seus efeitos e reflexos no desempenho físico.

\section{Efeitos da Privação de Sono}

As conseqüências das alterações no padrão de sono são diversas, podem ocorrer reduções na eficiência do processamento cognitivo, do tempo de reação e da responsividade atencional, além de prejuízo na memória, aumento da irrritabilidade, alterações metabólicas, endócrinas, imunológicas, quadros hipertensivos, cansaço, náuseas, dores de cabeça, ardência nos olhos, visão turva, dores articulares e diminuição da libido(32-35).

A falta de sono provoca prejuízos substanciais no desempenho físico e no cognitivo. As tarefas cognitivas complexas e as com um componente substancial de vigilância sofrem redução da eficiência do processamento cognitivo devido à privação de sono ${ }^{(36,37)}$. Além disso, essa condição resulta em uma lentificação do tempo de reação, uma menor vigilância, aumento nas distorções cognitivas e perceptuais ${ }^{(38)}$. Em testes mentais de aritmética após a privação de sono é observada uma lentificação da velocidade de cálculo e aumento no número de erros $^{(39)}$. O processamento de informações também é prejudicado, resultando em um aumento do tempo requerido para a tomada de decisões ${ }^{(40)}$. Martin(41) (1986) ainda sugeriu que os efeitos da privação de sono dependem do tipo e do tamanho da tarefa motora.

A recordação de informações verbais é substancialmente reduzida após uma noite de privação de sono ${ }^{(39)}$. Já durante a segunda noite de privação de sono os sujeitos só foram capazes de completar uma parte dos problemas resolvidos durante a avaliação basal(42).

Como já citado, a privação de sono causa prejuízo no estado de alerta, no desempenho cognitivo e no humor. A habilidade de realizar um trabalho mental declina 25\% para cada 24 horas sucessivas em que um indivíduo permanece acordado ${ }^{(43)}$. O prejuízo cognitivo observado com a privação de sono pode, em parte, ser evidenciado por um decréscimo no metabolismo cerebral de glicose, particularmente explicado pelo envolvimento de áreas frontais(44). 
De acordo com Horne ${ }^{(36)}$ e colaboradores (1983), em diversos estudos os resultados de testes de desempenho cognitivo não são consistentes, aparentemente até 36 a 40 horas de período de privação de sono, e muitas das vezes os efeitos são discretos, com uma quantidade significativa das pesquisas não demonstrando diferenças reais no desempenho com essa duração de privação de sono. Segundo os autores é possível que, os efeitos da motivação pessoal com o desempenho, possam reduzir ou superar os prejuízos na atenção e na concentração o que, de certa forma, representaria um resultado quase normal em muitos dos testes. É relevante ressaltar que essa afirmação é, no mínimo, questionável, uma vez que estudos com menor duração apresentam diferenças significativas no desempenho cognitivo devido à privação de sono e, embora muitos pesquisadores tenham tentado medir a motivação dos indivíduos durante os experimentos, muitos deles não observaram diferenças significativas quando se comparam os privados com os não-privados de sono.

A metaanálise conduzida por Pilcher e Huffcutt( ${ }^{(45)}$ (1996), mostrou que a maioria dos estudos confirma que os efeitos da privação de sono são respectivamente, mais pronunciados no humor, no desempenho cognitivo e motor. Segundo os autores, o humor e o desempenho cognitivo parecem ser mais sensíveis a privação parcial do sono do que à total de curta ou de longa duração. Uma possível explicação para esse fato se deve à alteração do efeito da ritimicidade circadiana no desempenho e no humor, observada na privação parcial de sono em contrapartida à interação dos ritmos biológicos observada na privação total de sono.

Outras conseqüências da privação de sono são: redução da responsividade atencional, declínio na habilidade de discriminar sinais, prejuízo de memória de curto prazo, alterações no humor (irrritabilidade, estado confusional, estresse e fadiga); diminuição de motivação e interesse; diminuição da capacidade de concentração; aumento de distrabilidade e erros por omissão; aumento de sonolência; alterações metabólicas, endócrinas e quadros hipertensivos ${ }^{(34-36,39,46,47)}$. Além disso, a privação de sono também prejudica a iniciação e a execução motora em resposta a um estímulo visual|(48)

As respostas bioquímicas durante a privação de sono ainda não estão bem estabelecidas, no entanto as evidências sugerem que ocorre um decréscimo da atividade tiróidiana ${ }^{(49)}$. Por outro lado, existe uma discordância quanto aos efeitos da privação em relação aos hormônios sexuais e adrenais, cortisol, adrenalina, catecolaminas, hematócrito, glicemia, creatinina e magnésio(50). Os hormônios como a noradrenalina, a prolactina, e o $\mathrm{GH}$, que exibem um comportamento circadiano em relação ao sono, parecem modificar esse padrão de periodicidade de excreção durante a perda de sono ${ }^{(51,52)}$.

Com o intuito de amenizar os efeitos deletérios da privação de sono, alguns autores têm focado seus trabalhos na verificação dos efeitos de cochilos durante o período da privação de sono. Estudando soldados que podiam dormir três horas por noite, Haslam ${ }^{(53)}$ (1985) observou que os soldados foram capazes de manter as suas atividades (mas não em um nível ótimo) durante os nove dias do estudo, enquanto que os que não tiveram permissão para dormir foram julgados militarmente não aptos após três dias. $O$ trabalho de Dingues ${ }^{(14)}$ e colaboradores (1987) demonstrou que a estratégia de cochilar por 2 horas, pode resultar em uma redução (mas não eliminação) do decréscimo observado na atenção e no desempenho, durante 54 horas de privação de sono. Naitoh(54) (1981) examinou o efeito de 45 horas de vigília sustentada, em que foi permitido dormir durante duas horas nas primeiras horas da manhã (4 às 6 horas) e após 53 horas de vigília sustentada em que foi permitido dormir por duas horas ao meio-dia. O autor descreveu que o cochilo realizado nas primeiras horas do dia resultava em uma prolongada e marcante inércia do sono, não tendo nenhuma ação recuperativa. Por outro lado, o cochilo ao meio-dia produziu uma curta inércia do sono e teve um efeito nitidamente recuperativo. Naitoh concluiu que o poder de recuperação de um cochilo depende não apenas da sua duração, mas principalmente de quanto tempo de vigília o sujeito apresenta e da hora do dia em que o mesmo foi realizado.

No entanto sugere-se cautela na interpretação dos efeitos da privação de sono, devendo-se considerar as diversas variáveis norteadoras, em particular o gênero e a idade(55).

\section{Privação do Sono e Exercício Físico}

Muitos atletas têm a visão de que a perda ou interrupção do sono é um fator que contribui para o seu fracasso no esporte. A maioria dos estudos que investigaram os efeitos da privação do sono no desempenho físico, focaram primariamente, seus efeitos no desempenho aeróbio submáximo(56-59) e no consumo máximo de oxigênio(58,60,61). Outras pesquisas examinaram as respostas neurológicas ${ }^{(62)}$ e a força anaeróbia de indivíduos privados de sono ${ }^{(57,58,60)}$

Quando se considera os efeitos da privação de sono no desempenho aeróbio, muitos indicadores fisiológicos e de desempenho são considerados. Os trabalhos que envolveram um período de privação de 30 horas $^{(63,64)}$ até 60 horas $^{(59)}$ não encontraram modificações evidentes. No entanto, o estudo de Bond e colaboradores ${ }^{(57)}$ (1986) encontrou diferenças menores em medidas de ventilação, $\mathrm{VCO}_{2}$ e VO $\mathrm{CO}_{2}$ nos sujeitos privados de sono por 42 horas, quando comparados aos controles nãoprivados, sugerindo, que para uma eficiência máxima do exercício em intensidades acima de $75 \%$ do $\mathrm{VO}_{2}$ máx deveria ser precedido por uma boa noite de sono. A partir desse trabalho houve um consenso entre os pesquisadores de que a privação de sono seria capaz de provocar um pequeno efeito ou até mesmo nenhum, no desempenho aeróbio.

Mougin e colaboradores ${ }^{(61)}$ (1991) notaram que, embora fosse possível sustentar a mesma intensidade máxima de exercício, os sujeitos que tiveram seu sono interrompido por um período de três horas exibiram um menor VO2 máx., uma alta ventilação máxima e submáxima, maiores valores da freqüência cardíaca e do lactato sanguíneo.

Com o intuito de investigar o efeito de três noites de privação de sono no consumo de oxigênio, na eficiência mecânica e no exercício de carga constante e estável, Horne e Pettit ${ }^{(65)}$ (1984) observaram sete sujeitos que pedalaram em uma bicicleta ergométrica, diariamente das 4 às 16 horas, utilizando como carga de trabalho 40, 60 e $80 \%$ do $\mathrm{VO}_{2}$ máx., e um grupo controle idêntico com exceção da privação de sono. A eficiência mecânica apresentou uma grande variabilidade durante a privação de sono, mas os efeitos do treinamento foram similares para ambos os grupos, concluindo que, a habilidade fisiológica para realizar um trabalho com o tipo e a duração do protocolo utilizado, não era adversamente afetada após 72 horas de privação de sono.

Vondra e colaboradores ${ }^{(66)}$ (1981) encontraram redução significativa na atividade de enzimas do metabolismo energético na musculatura esquelética após 120 horas de privação de sono, indicando uma diminuição da capacidade oxidativa aeróbia, uma redução da função de transportar equivalentes reduzidos do citosol através da membrana mitocondrial, uma acentuação relativa da atividade glicolítica anaeróbia e um metabolismo muscular do tipo pré-diabético.

Embora haja certo consenso do não efeito da privação de sono no desempenho aeróbio, é importante ressaltar que, durante a privação de sono, ocorre um aumento significativo na percepção do esforço (57-59,63) e uma redução do tempo para a exaustão, sendo que esses parâmetros afetam o desempenho aeróbio. A percepção ao esforço é um efeito psicológico em que os sujeitos descrevem uma maior resistência em relação ao desempenho de uma tarefa, com as mesmas exigências fisio- 
lógicas após a privação de sono ${ }^{(64)}$. Martin ${ }^{(67)}$ (1981) observou aumento na percepção de esforço em sujeitos privados de sono por 36 horas, quando comparados a controles, sugerindo que o efeito psicológico da perda de sono aguda poderia contribuir para o decréscimo da tolerância ao exercício pesado prolongado.

Engle-Friedman e colaboradores ${ }^{(68)}$ (2003) notaram que o aumento da sonolência, da fadiga e do tempo de reação observado com a privação de sono, está associado à escolha de tarefas menos difíceis, sendo que os sujeitos privados, quando comparados aos com controles, não percebem essa redução do esforço. Esse estudo demonstrou que a perda de sono resulta em um comportamento de escolha de baixo esforço para ajudar a manter a acurácia da resposta a uma tarefa.

Empiricamente, o exercício físico é usado para combater a sonolência quando uma situação prolongada de vigília precisa ser mantida, inconscientemente este pressuposto empírico adota o efeito da produção de calor como elemento para promover a vigília. Neste contexto, Matsumoto e colaboradores ${ }^{(69)}$ (2002) alertaram para a possibilidade de que o aumento do exercício físico durante a vigília prolongada poderia ampliar a dissociação entre a avaliação subjetiva da sonolência e a função cerebral atual, resultando no aumento do risco de erro humano pela possibilidade de "mascarar" um possível prejuízo da função cerebral.

Quanto ao comportamento da freqüência cardíaca nos exercícios aeróbios durante a privação de sono, ainda restam diversas questões a serem respondidas. Martin \& Chen ${ }^{(56)}$ (1984) e Savis ${ }^{(70)}$ (1994) não encontraram flutuações significativas na freqüência cardíaca durante um período prolongado de privação de sono. No entanto, outros estudos reportaram uma clara redução da freqüência cardíaca em diferentes cargas de trabalho após a privação do sono(57,59,64). Para Martin \& Gaddis(63) (1981), ocorre uma redução significativa da freqüência cardíaca nas cargas máximas, embora ocorra uma pequena ou nenhuma mudança nos níveis submáximos.

Para participantes de eventos multieportivos de longa duração, Scott \& McNaughton ${ }^{(71)}$ (2003) sugeriram que, dentro de uma perspectiva fisiológica, a perda de sono pode não ser considerada como um fator limitante para o desempenho, porém, sujeitos privados de sono apresentam uma redução da tolerância a esforços prolongados ${ }^{(72)}$. VanHelder \& Radomski(58) (1989) sugeriram que a significativa redução da tolerância à glicose, após um período de privação de sono, pode ser um dos mecanismos responsáveis pelas implicações em sustentar o exercício aeróbio.

Poucos trabalhos têm sido conduzidos na investigação dos efeitos da privação de sono sobre a potência anaeróbia, força e respostas eletro-mecânicas, resultando assim em uma indefinição quanto aos resultados. Souissi e colaboradores ${ }^{(73)}$ (2003) observaram que o desempenho anaeróbio não foi afetado após 24 horas de vigília sustentada. No entanto, observaram modificações após 36 horas sem dormir. Por outro lado, alguns trabalhos sugerem que a privação de sono não possui efeitos na força e na potência máxima ${ }^{(58,74)}$, na contração isométrica máxima ${ }^{(62)}$, na fadiga muscular e no trabalho total( ${ }^{(74)}$. Considerando o fato de que durante a privação de sono ocorre o aumento na percepção ao esforço, esses resultados sugerem que, em relação à força, sujeitos privados de sono por 60 horas podem reagir rápido com a mesma força de um sujeito que dormiu sete horas de sono à noite ${ }^{(62)}$.

Takeuchi e colaboradores ${ }^{(75)}$ (1985) demonstraram que 64 horas de privação de sono não prejudicam a força de preensão manual ou o pico de torque para a extensão da perna, mas, pode prejudicar o salto vertical e a extensão de joelho nas baixas velocidades. Já Bulbulian e colaboradores $^{(76)}$ (1996), reportaram que a privação de sono por um período de 30 horas era capaz de afetar o pico de torque, mas não o índice de fadiga.
Para examinar a efetividade do exercício no desempenho sustentado, LeDuk e colaboradores ${ }^{(77)}$ (2000) investigaram 12 voluntários que fizeram exercício em esteira a $70 \%$ do $\mathrm{VO}_{2}$ máx., por 10 minutos a cada duas horas, durante um período de 40 horas de privação de sono. Os voluntários se mostraram mais alertas após o exercício e o EEG revelou que o exercício aumentou a atividade das ondas lentas (delta e teta) e rápidas (alfa e beta). Os autores concluíram que, curtos acréscimos de exercícios físicos, podem melhorar o aumento da sonolência e da fadiga associado à privação de sono por um curto período de tempo, mas não podem prevenir o decréscimo do desempenho. Aumentos significativos da fadiga e da sonolência podem ocorrer se o exercício tiver uma duração inferior à uma hora.

A privação de sono também é capaz de promover mudanças nos ajustes termorregulatórios durante o exercício. Sawka e colaboradores $^{(78)}$ (1984) investigaram uma possível mudança no "set-point" da temperatura corporal, e modificações na taxa de sudorese e na condutância térmica em sujeitos privados de sono submetidos a um protocolo de exercício físico de intensidade moderada. Eles observaram que a privação de sono alterou a magnitude da resposta da taxa de sudorese ao aumento da temperatura corporal durante o exercício, encontrando uma redução total da taxa de sudorese corporal e da do peito no final do exercício de 27 e 19\%, respectivamente. Os autores especularam que a privação poderia ter alterado os níveis de monoaminas na região do hipotálamo, modificando assim o controle central da termorregulação. Uma outra possibilidade levantada pelos autores foi uma possível alteração do sinal efetor após o seu início no hipotálamo, o que resultaria na dessincronização observada na taxa de sudorese do peito e redução na resposta do suor. Ainda nesta temática, Kolka e Stepheson ${ }^{(79)}$ (1988) observaram que a vasodilatação reflexa cutânea durante o exercício parece estar reduzida, tanto por fatores locais quanto centrais, após 33 horas de vigília sustentada.

Os reais efeitos da privação de sono no desempenho físico ou fisiológico são conflitantes e/ou inconclusivos. As razões para essas disparidades podem ser explicadas pelos diferentes tipos, freqüência, intensidade e duração dos exercícios empregados, assim como por outros parâmetros, entre os quais, o tempo de privação e a idade dos sujeitos experimentais.

\section{Exercício de Longa Duração e Privação de Sono}

Estudos envolvendo exercício físico prolongado e privação de sono se tornaram nos últimos anos uma rica linha de pesquisa, em parte isso se deve a possibilidade de acompanhamento de uma possível interação desses dois parâmetros e pelo crescimento de modalidades multiesportivas que em sua essencialidade expõem o atleta a condição de privação de sono.

Até recentemente, os resultados sobre a atividade prolongada e a privação de sono eram oriundos de pesquisas militares, atualmente, as pesquisas têm enfocado os atletas de elite que participam das provas de ultraendurance.

O Race Across América (RAAM) é um exemplo disso, trata-se de uma prova em que os atletas pedalando sozinhos cruzam os Estados Unidos percorrendo um total de 2900 milhas. Diferente do Tour de France, nesta prova, não existem paradas para descanso. Para vencer essa prova, o atleta leva aproximadamente oito dias. Smith e colaboradores ${ }^{(80)}(1998)$ estudaram sete atletas e relataram que o vencedor dormia aproximadamente apenas duas horas por dia, concluindo que alguns atletas apresentavam uma habilidade ímpar para manter o desempenho e vencer apesar da privação de sono.

Em 1990, Edinger e colaboradores(81) acompanharam dois tenistas por 146 horas durante um jogo em que era permitido dormir 
por um período de 4 a 5 horas por noite. Ao final do jogo, os atletas apresentaram declínios na memória e na capacidade de codificação percepto-motora. Ao longo dos sets os atletas relataram aumento de fadiga, sendo que um deles sofreu lesões e desenvolveu desorientação intermitente no sexto dia.

O sono, como a muitas outras necessidades, tem sido constantemente priorizado para um desempenho físico ótimo. No Eco-Challenge de 2002 (uma conhecida corrida de aventura), a equipe vencedora completou a prova em aproximadamente 170 horas, e durante esse período os membros da equipe dormiram apenas 17 horas. Isso representou um grande avanço comparado à prova de 2001, em que a equipe líder desenvolveu uma extrema fadiga física e cognitiva. A estratégia para a prova de 2002 foi a de não passar mais que 30 horas sem dormir, ao contrário da de 2001 que era dormir apenas se fosse extremamente necessário(82).

Nosso grupo investigou a arquitetura do sono de atletas de corrida de aventura que participaram de três edições da ECOMOTION-PRO, uma corrida aventura longa onde os atletas têm que percorrer entre 450 a $550 \mathrm{~km}$ em diferentes modalidades esportivas e que durante essa prova experimentam a condição de privação de sono. Quando comparamos a arquitetura do sono dos atletas (basal versus pós-prova), observamos modificações importantes nos estágios do sono, sendo o rebote de sono delta e o rebote em um período subseqüente do sono REM, os resultados mais expressivos. Além disso, esse estudo também permitiu inferir que essas alterações parecem estar implicadas com o volume de exercício e com as possíveis alterações decorrentes do contexto da prova ${ }^{(83,84)}$.

Outros trabalhos que investigaram os efeitos da privação de sono e a sua relação com o exercício físico observaram atletas de regatas na categoria solo. Ellen MacArthur, uma competidora do 2001 Vendee Globe Sailing Race, praticou o "Cluster-napping" durante uma prova solo de 25.000 milhas. Essa técnica foi sugerida pelo pesquisador Stampi ${ }^{(85)}$ (1990) como um programa de alerta. A duração do sono variou de acordo com as condições do ambiente e foi geralmente realizada por cochilos diurnos, a estratégia era fragmentar o sono em cochilos com a duração entre 25 a 40 minutos cada, permitindo assim uma rápida análise dos equipamentos e das condições do tempo quando a atleta acordava. Os cochilos da atleta duraram em média 36 minutos, sendo o tempo total de sono diário em torno de 5,5 horas durante os 94 dias da prova. A atleta fez o melhor tempo para uma mulher e chegou muito próxima do segundo lugar geral. Um trabalho semelhante foi realizado por Bennet ${ }^{(86)}$ (1973) que observou 19 navegadores solitários em uma prova de 3.000 milhas, embora a média de sono não tenha sido relatada nesse estudo, os autores descreveram que os erros foram bastante comuns em situações adversas, particularmente, quando havia problemas com o barco, também foram notadas ilusões ou alucinações visuais, auditivas e olfatórias.

\section{CONCLUSÃO}

Em uma perspectiva fisiológica, a presente revisão aponta que a privação de sono associado ao exercício físico não poderia ser considerada como um fator limitante do desempenho atlético, particularmente na atividade aeróbia. Em vista dos resultados, há de fato uma grande similaridade com outros estudos que conduziram investigações sobre os efeitos do exercício físico no padrão de sono, mas sem a concomitante privação de sono(87-91), esta similaridade sugere uma interação entre a privação de sono e o exercício físico, onde por mecanismos ainda não esclarecidos, o exercício físico poderia "proteger" o organismo quando a privação de sono ocorre simultaneamente. Esse ponto parece ser importante principalmente quando consideramos o exercício como uma alternativa para reverter os efeitos deletérios da privação de sono, mas é importante mencionar que o exercício físico por aumentar o estado de alerta e liberar diferentes substâncias neuroquímicas durante a sua realização, poderia mascarar os efeitos da privação de sono. Cabe aqui a reflexão de que esse "mascaramento" pode ser confundido com o efeito protetor, e isso pode ser bastante efetivo principalmente quando considerado o aumento observado na percepção de esforço com a privação de sono, que por si só representa um importante fator para diminuir o desempenho atlético e comprometer o resultado final de um atleta. Novos estudos que envolvam essa temática são desejáveis, entretanto é válido mencionar a grande dificuldade de realizar um protocolo experimental dessa natureza, principalmente utilizando voluntários sem nenhum tipo de compensação financeira.

\section{AGRADECIMENTOS}

Os autores são gratos a Associação Fundo de Incentivo à Psicofarmacologia (AFIP); CAPES; ao Instituto do Sono; ao CEPE; e a FAPESP/ CEPID (\#98/14303 S.T).

Todos os autores declararam não haver qualquer potencial conflito de interesses referente a este artigo.

\section{REFERÊNCIAS BIBLIOGRÁFICAS}

1. Roeths T, Roth T. Sleep-wake state and memory function. Sleep. 2000; 23: S64-8.

2. Aserinsky E, Kleitman N. Regulary occurring periods of eye motility and concomitant phenomena during sleep. Science. 1953; 118: 273-4.

3. Dement W, Kleitman N. Cyclic variations in EEG during sleep and their relation to eye movements, body motility, and dreaming. Eletroencephalogr Clin Neurophysiol Suppl. 1957; 9: 673-90.

4. Rechtschaffen A, Kales A. A Manual of standardized terminology, techniques, and scoring system for sleep stages of human subjects. Los Angeles: Brain Information Service/Brain Research Institute/UCLA; 1968: 57.

5. Martins PJF, Mello MT, Tufik S. Exercício e sono. Rev Bras Med Esporte. 2001; 7: 28-36.

6. Chen W, Kushida CA. Perspectives. In: Sleep deprivation: basic science, physiology and behavior. New York: Marcel Dekker. 2005: 1-30.

7. De Manacéine M. Quelques observations experimentales sur I 'influence de l'insomie absolute. Arch Ital Biol. 1894; 21:332-5.

8. Bentivoglio M, Grassi-Zucconi G. The pioneering experimental studies on sleep deprivation. Sleep. 1997; 20: 570-6.

9. Patrick GTW, Gilbert JA. On the effects of loss of sleep. Psychol Rev 1896; 3: 469-83.

10. Kleitman N. Studies on the physiology of sleep. V. Some experiments on puppies. Am J Physiol. 1920; 84: 386-95.

11. Williams HL, Lubin A, Goodnow JJ. Impaired performance with acute sleep loss. Psychol Monogr. 1959; $73: 1-26$

12. Gulevich G, Dement W, Johnson L. Psychiatric and EEG observations on a case of prolonged (264 hours) wakefulness. Arch Gen Psychiatry. 1966; 15: 29-35.

13. Dement W. The effect of dream deprivation. Science. 1960; 131: 1705-7.

14. Dinges DF, Orne MT, Whitehouse WG, Orne EC. Temporal placement of a nap for alertness: contributions of circadian phase and prior wakefulness. Sleep. 1987; 10: 313-29.

15. Siegel J M. Clues to the functions of mammalian sleep. Nature. 2005; 437: 1264-71.

16. Tufik S, Lindsey CJ, Carlini EA. Does REM sleep deprivation induce a supersensitivity of dopaminergic receptors in the rat brain? Pharmacology. 1978; 16: 98-105.

17. Tufik S. Changes of response to dopaminergic drugs in rats submitted to REM-sleep deprivation. Psychopharmacology (Berl). 1981a; 72: 257-60.

18. Tufik S. Increased responsiveness to apomorphine after REM sleep deprivation: supersensitivity of dopamine receptors or increase in dopamine turnover? J Pharm Pharmacol. 1981 b; 33: 732-8.

19. Nunes Junior GP, Tufik S, Nobrega JN. Autoradiographic analysis of D1 and D2 dopaminergic receptors in rat brain after paradoxical sleep deprivation. Brain Res Bull. 1994a; 34: 453-6.

20. Nunes Junior GP, Tufik S, Nobrega JN. Decreased muscarinic receptor binding in rat brain after paradoxical sleep deprivation: an autoradiographic study. Brain Res. 1994b; 645: 247-52.

21. Troncone LR, Ferreira TM, Braz S, Silveira Filho NG, Tufik S. Reversal of the increase in apomorphine- 
induced stereotypy and aggression in REM sleep deprived rats by dopamine agonist pretreatments. Psychopharmacology (Berl). 1988; 94: 79-83.

22. Neumann BG, Troncone LR, Braz S, Tufik S. Modifications on dopaminergic and cholinergic systems induced by the water tank technique: analysis through yawning behavior. Arch Int Pharmacodyn Ther. 1990; 308: 32-8.

23. Troncone $L R$, Tufik $S$. Effects of selective adrenoceptor agonists and antagonists on aggressive behavior elicited by apomorphine, DL-dopa and fusaric acid in REM-sleep-deprived rats. Physiol Behav. 1991; 50: 173-8.

24. D'Almeida V, Hipolide DC, Azzalis LA, Lobo LL, Junqueira VB, Tufik S. Absence of oxidative stress following paradoxical sleep deprivation in rats. Neurosci Lett. 1997; 235: 25-8.

25. D'Almeida V, Lobo LL, Hipolide DC, de Oliveira AC, Nobrega JN, Tufik S. Sleep deprivation induces brain region-specific decreases in glutathione levels. Neuroreport. 1998; 9: 2853-6.

26. Suchecki D, Lobo LL, Hipolide DC, Tufik S. Increased ACTH and corticosterone secretion induced by different methods of paradoxical sleep deprivation. J Sleep Res. 1998; 7: 276-81.

27. Hipolide DC, Suchecki D, de Carvalho Pinto AP, Chiconelli Faria E, Tufik S, Luz J. Paradoxical sleep deprivation and sleep recovery: effects on the hypothalamic-pituitary-adrenal axis activity, energy balance and body composition of rats. J Neuroendocrinol. 2006; 18: 231-8.

28. Palma BD, Gabriel A Jr, Bignotto M, Tufik S. Paradoxical sleep deprivation increases plasma endothelin levels. Braz J Med Biol Res. 2002; 35: 75-9.

29. Bueno OF, Lobo LL, Oliveira MG, Gugliano EB, Pomarico AC, Tufik S. Dissociated paradoxical sleep deprivation effects on inhibitory avoidance and conditioned fear. Physiol Behav. 1994; 56: 775-9.

30. Godoi FR, Oliveira MG, Tufik S. Effects of paradoxical sleep deprivation on the performance of rats in a model of visual attention. Behav Brain Res. 2005; 165: 138-45.

31. Andersen ML, D'Almeida V, Martins PJ, Antunes HK, Tufik S. Effects of paradoxical sleep deprivation and cocaine on genital reflexes in hyperlipidic-fed rats. Pharmacol Biochem Behav. 2005; 81: 758-63.

32. Shephard RJ, Shek PN. Interactions between sleep, other body rhythms, immune responses, and exercise. Can J Appl Physiol. 1997; 22: 95-116.

33. Spiegel $K$, Leprolt $R$, Van Cauter E. Impact of sleep debt on metabolic and endocrine function. Lancet. 1999; 354: 1435-9.

34. Brouwers FM, Lenders JW. Sleep-disordered breathing and hypertension. N Engl J Med. 2000; 343: 967.

35. Bonnet MH, Arand DL. Clinical effects of sleep fragmentation versus sleep deprivation. Sleep Med Rev. 2003; 7: 297-310

36. Horne JA, Anderson NR, Wilkinson RT. Effects of sleep on signal detection measures of vigilance: implications for sleep function. Sleep. 1983; 6: 347-58.

37. Ellenbogen JM. Cognitive benefits of sleep and their loss due to sleep deprivation. Neurology. 2005; 64: E25-7.

38. Krueger GP. Sustained work, fatigue, sleep loss and performance: a review of the issues. Work Stress. 1989; 3: 129-41.

39. Webb WB, Levy CM. Effects of spaced and repeated total sleep deprivation. Ergonomics. 1984; 27: 45-58.

40. Asken MJ, Raham DC. Resident performance and sleep deprivation: a review. J Med Educ. 1983; 58: 382-8.

41. Martin BJ. Sleep deprivation and exercise. Exerc Sport Sci Rev. 1986; 14: 213-29.

42. Carskadon MA, Dement WC. Effects of total sleep loss on sleep tendency. Percept Mot Skills. 1979; 48: 495-506.

43. Belensky G, Penetar DM, Thorne D, Popp K, John L, Thomas M, Sing H, Balkin T, Wesensten N, Redmond D. The effects of sleep deprivation on performance during continuous combat operations. In: National Academy Press. Food Components to enhance performance. 1994; 127-35.

44. Thomas M, Sing H, Belenky G, Thorne D, Balkin T, Penetar D et al. Positron emission tomography measurements of human regional cerebral glucose wtilization following 48 hours of sleep deprivation. Soc Neurosci Abstracts. 1988; 14: 48. [Presented at 18 Annual Meeting of the Society for Neuroscience; 1988 november 13-18; Toronto, Canada].

45. Pilcher J, Huffcutt Al. Effects of sleep deprivation on performance: a meta-analysis. Sleep. 1996; 19: 318-26.

46. Bonnet MH. Effect of sleep disruption on sleep, performance, and mood. Sleep. 1985; 8: 11-9.

47. Harrison Y, Horne JA, Rothwell A. Prefrontal neuropsychological effects of sleep deprivation in young adults-a model for healthy aging? Sleep. 2000; 23: 1067-73.

48. Sanders AF, Reitsma WD. The effect of sleep-loss on processing information in the functional visual field. Acta Psychol. 1982; 51: 149-62.

49. Gary KA, Winokur A, Douglas SD, Kapoor S, Zaugg L, Dinges DF. Total sleep deprivation and the thyroid axis: effects of sleep and waking activity. Aviat Space Environ Med. 1996; 67: 513-9.

50. Beck U. Hormonal secretion during sleep in man. Modification of growth hormone and prolactin secretion by interruption and selective deprivation of sleep. Int J Neurol. 1981; 15: 17-29.

51. Akerstedt T. Altered sleep-wake patterns and circadian rhythms. Acta Physiol Scand Suppl. 1979; 469: 1-48.

52. Parker DC, Rossman LG, Kripke DF, Hershman JM, Gibson W, Davis C et al. Endocrine rhythms across sleep-wake cycles in normal young men under basal conditions. In: Orem J, Barnes CD, editors. Physiology in sleep. New York: Academic, 1980: 145-79.

53. Haslam DR. Sustained operations and military performance. Behav Res Methods Instrum Comput. 1985; 17: 9-95

54. Naitoh P. Circadian cycles and restorative power of naps. In: Johson LC, Tepas DI, Colquhoum WP, Colligan MJ eds Biological rhythms, sleep and shift work. Advances in Sleep Research, Vol 7 New York: 1981: 553-80.

55. Corsi-Cabrera M, Sanchez Al, Del-Río-Portilha Y, Villanueva Y, Pérez-Garci E. Effect of 38 h of total sleep deprivation on the waking EEG in women: sex differences. Int J Psychophysiol. 2003; 50(3): 213-24.

56. Martin BJ, Chen HI. Sleep loss and the sympathoadrenal response to exercise. Med Sci Sports Exerc. 1984; 16: 56-9.

57. Bond V, Balkissoon B, Franks BD, Brownlow R, Caprarola M, Bartley D et al. Effects of sleep deprivation on performance during submaximal and maximal exercise. J Sports Med Phys Fitness 1986; 26: 169-74.

58. VanHelder T, Radomski MW. Sleep deprivation and the effect on exercise performance. Sports Med 1989; 7: 235-47.

59. Montelpare WJ, Plyley MJ, Shephard RJ. Evaluating the influence of sleep deprivation upon circadian rhythms of exercise metabolism. Can J Sport Sci. 1992; 17: 94-7.

60. Webb WB, Kaufmann DA, Devy CM. Sleep deprivation and physical fitness in young and older subjects. J Sports Med Phys Fitness. 1981; 21: 198-202.

61. Mougin F, Simon-Rigaud ML, Davenne D, Renaud A, Garnier A, Kantelip JP et al. Effects of sleep disturbances on subsequent physical performance. Eur J Appl Physiol Occup Physiol. 1991; 63: 77-82.

62. Symons JD, Bell DG, Pope J, VanHelder T, Myles WS. Elestromechanical response times and muscle strength after sleep deprivation. Can J Sport Sci. 1988; 13: 225-30.

63. Martin BJ, Gaddis GM. Exercise after sleep deprivation. Med Sci Sports Exerc. 1981; 13: 220-3.

64. Martin B, Hanney R. Self-selected exercise intensity is unchanged by sleep loss. Eur J Appl Physiol Occup Physiol. 1982; 49: 79-86.

65. Horne JA, Pettitt AN. Sleep deprivation and the physiological response to exercise under steady-state conditions in untrained subjects. Sleep. 1984; 7: 168-79.

66. Vondra K, Brodan V, Bass A, Kuhn E, Teisinger J, Andel M et al. Effects of sleep deprivation on the activity of selected metabolic enzymes in skeletal muscle. Eur J Appl Physiol Occup Physiol. 1981; 47: 41-6.

67. Martin BJ. Effect of sleep deprivation on tolerance of prolonged exercise. Eur J Appl Physiol Occup Physiol. 1981; 47: 345-54.

68. Engle-Friedman M, Riela S, Golan R, Ventuneac AM, Davis CM, Jefferson AD et al. The effects of sleep loss on next day effort. J Sleep Res. 2003; 12:113-24.

69. Matsumoto Y, Mishima K, Satoh K, Shimizu T, Hishikawa Y. Physical activity increases the dissociation between subjective sleepiness and objective performance levels during extended wakefulness in human. Neurosci Lett. 2002; 326: 133-6.

70. Savis J. Sleep and athletic performance: overview and implications for sport psychology. The Sports Psychologist. 1994; 8: 111-25.

71. Scott JPR, McNaughton LR. Sleep deprivation, energy expenditure and cardiorespiratory function. Int J Sports Med. 2004; 25: 421-6.

72. Martin BJ, Bender PR, Chen H. Stress hormonal response to exercise after sleep loss. Eur J Appl Physiol Occup Physiol. 1986; 55: 210-4.

73. Souissi N, Sesboüé B, Gauthier A, Larue J, Davenne D. Effects of one night's sleep deprivation on anaerobic performance the following day. Eur J Appl Physiol. 2003; 89: 359-66.

74. Bond V, Kresham K, Balkissoon B, Tuckson L, Caprarola M, Johnson D et al. Effects of sleep deprivation on muscle function during an isokinetic contraction. J Sports Med Phys Fitness 1988; 28: 1-6.

75. Takeuchi L, Davis GM, Plyley M, Goode R, Shephard RJ. Sleep deprivation, chronic exercise and muscular performance. Ergonomics. 1985; 28: 591-601.

76. Bulbulian R, Heaney JH, Leake CN, Sucec AA, Sjoholm NT. The effects of sleep deprivation and exercise load on isokinetic leg strength and endurance. Eur J Appl Physiol Occup Physiol 1996; 73: 273-7.

77. LeDuck PA, Caldwell Jr JA, Ruyak PS. The effects of exercise as a countermeasure for fatigue in sleepdeprived aviators. Mil Psychol. 2000; 12: 249-66.

78. Sawka MN, Gonzalez RR, Pandolf KB. Effects of sleep deprivation on thermoregulation during exercise. Am J Physiol Regul Integr Comp Physiol. 1984; 246: R72-7.

79. Kolka MA, Stephenson LA. Exercise thermoregulation after prolonged wakefulness. J Appl Physiol. 1988; 64: 1575-9.

80. Smith RS, Walsh JS, Dement WC. Sleep deprivation and the Race Across America (RAAM). Sleep. 1998; 22: $\mathrm{S303.}$

81. Edinger JD, Marsh GR, McCall WV, Erwin CW, Lininger AW. Daytime functioning and nighttime sleep before, during, and after a 146-hour tennis match. Sleep. 1990; 13: 526-32.

82. Smith RS, Reily TP. Athletic Performance. In: Sleep Deprivation. Clinical Issues, Pharmacology and Sleep Loss Efects. Kushida CA editor. New Your: 2005: 313-34.

83. Antunes, HKM; Silva RS; Bittencourt, LRA; De Mello, MT; Tuffik, S. Sleep patterns of athletes after chronic sleep deprivation and sustained exercise. Sleep 2005; 28: A142.

84. Antunes, HKM. A influência do exercício físico e da privação de sono no padrão de sono em atletas de corrida de aventura. [Tese de Doutorado], Universidade Federal de São Paulo, UNIFESP, 2006.

85. Stampi C. Cochilos e padrões polifásicos do sono humano. In: Reimão R. Sono: Aspectos Atuais. São Paulo: Atheneu, 1990: 249-75.

86. Bennet G. Medical and psychological problems in the 1972 singlehanded transatlantic yacht race. Lancet. 1973; 2: 747-54.

87. Horne JA. The effects of exercise upon sleep: a critical review. Biol Psychol. 1981; 12: 241-90.

88. Driver HS, Meintjes AF, Rogers GG, Shapiro CM. Submaximal exercise effects on sleep patterns in young women before and after an aerobic training programme. Acta Physiol Scand Suppl. 1988; 574: 8-13.

89. O'Connor PJ, Youngstedt SD. Influence of exercise on human sleep. Exerc Sport Sci Rev. 1995; 23: 105-34.

90. Driver HS, Taylor SR. Exercise and sleep. Sleep Med Rev. 2000; 4: 387-402.

91. Youngstedt SD. Effects of exercise on sleep. Clin Sports Med. 2005; 24: 355-65. 